\title{
INJERTO AUTÓLOGO
}

\section{Sánchez González, V. Vicent Carsí, E. Navarrete Faubel}

Unidad de Pie y Tobillo. Servicio de Cirugía Ortopédica y Traumatología.

Hospital Universitari i Politècnic La Fe de Valencia

\section{Introducción}

A pesar de la aparición y el desarrollo en las últimas décadas de los denominados sustitutos óseos ${ }^{(1-3)}$, la utilización del injerto óseo autólogo (obtenido del propio paciente) continúa siendo aceptada como patrón oro (gold standard) en aquellos casos en los que necesitamos aumentar los índices de consolidación en los procedimientos quirúrgicos sobre pie y tobillo.

Históricamente, el injerto óseo autólogo se ha estado utilizando en múltiples cirugías desde $1944^{(4)}$, inicialmente para rellenar defectos óseos. Desde entonces, sus indicaciones han ido en aumento de forma exponencial. Sin embargo, su uso en las diferentes cirugías no es garantía absoluta de éxito. La incorporación del injerto autólogo depende de varios factores, entre los que se incluyen un contacto estrecho entre las superficies óseas viables, la adecuada vascularización del lecho receptor, la estabilidad mecánica, el proceso del remodelado óseo y el número de células en el sitio receptor ${ }^{(5)}$. Además, la utilización de injerto óseo autólogo está restringida por la morbilidad asociada al sitio donante, el aumento en los tiempos quirúrgicos y su cantidad limitada. Por todo esto, la tendencia actual es reducir el aporte de injerto autólogo en muchas cirugías en las que clásicamente era usado y utilizar sustitutos diferentes al hueso autólogo. Sin embargo, esta elección no debería basarse solo en la preferencia del cirujano o en consideraciones biológicas o biomecánicas de la sustancia a emplear, sino también en la evidencia científica disponible en la literatura.

Pero, tal vez, lo más destacable es que apenas existen estudios prospectivos, aleatorizados, que comparen directamente los resultados obtenidos en las diferentes cirugías de pie y tobillo llevadas a cabo con injerto autólogo o sin él. De hecho, el uso de injerto autólogo solo tiene grado de recomendación C en algunos estudios recientes, lo que corresponde a nivel IV y V de evidencia científica( ${ }^{(6)}$. Esto nos hace

FS $\odot 2017$ SEMCPT. Publicado por Imaidea Interactiva en FONDOSCIENCE ${ }^{\circledR}$ (www.fondoscience.com). 
pensar que hemos estado utilizando históricamente injerto de hueso autólogo solo basado en teorías empíricas no demostradas ${ }^{(7)}$.

El objetivo de este artículo es llevar a cabo una actualización del tema del uso de injerto autólogo óseo en cirugía de pie y tobillo, mediante una revisión bibliográfica y, tal vez, poder sentar unas recomendaciones con la evidencia científica suficiente.

\section{¿Qué dice la literatura?}

\section{Indicaciones}

El injerto autólogo de hueso se utiliza habitualmente en gran cantidad de cirugías que implican al pie y el tobillo con el fin de solucionar numerosas patologías (artropatías degenerativas y deformantes, traumatismos, necrosis avascular, tumores...) para mejorar las tasas de consolidación en las artrodesis, osteotomías y pseudoartrosis o ausencias de consolidación.

De acuerdo con lo disponible en la literatu$\mathrm{ra}^{(8)}$, las intervenciones quirúrgicas en las que se utiliza con más frecuencia injerto óseo autólogo son las artrodesis. Dentro de estas (por orden de frecuencia) están: artrodesis tibiotalar, subtalar, tibiotalocalcánea y triple tarsiana. En menor frecuencia, tibiocalcánea, talonavicular, calcaneocuboidea, naviculocuneiforme, tarsometatarsal, metatarsofalángicas o incluso interfalángicas ${ }^{(9)}$.

Además, también se aporta injerto óseo autólogo en osteotomías calcáneas (Evans o de alargamiento calcáneo)(10) o cuneanas (Cotton o similares), como relleno de cavidades (quistes) o defectos óseos, necrosis avasculares y revisión quirúrgica de las ausencias de consolidación.

\section{Objetivo: ¿es necesario?}

Según el artículo publicado por Valderrábano(11), el retropié tiene un ambiente difícil para la consolidación ósea, debido a la carga mecánica, la aportación vascular restringida y la escasa cobertura de pares blandas. Un 14\% de los pacientes a los cuales se les realiza una artrodesis del retropié o una osteotomía desarrollará una pseudoartrosis. Este porcentaje aumenta en aquellos con factores de riesgo para la consolidación ósea, como diabéticos o fumadores ${ }^{(12)}$. Hay estudios que comunican porcentajes de ausencia de consolidación cercanos al $40 \%$ en esta población de riesgo ${ }^{(13-15)}$. Parece lógico, pues, el aporte de injerto autólogo para aumentar el porcentaje de consolidación en pacientes con factores de riesgo.

\section{Ventajas}

El injerto autólogo es, hoy por hoy, el único material conocido capaz de ser osteoconductor, osteoinductor y con propiedades osteogénicas ${ }^{(5)}$. Además, no existe riesgo de transmisión infecciosa, como ocurre con aloinjertos o xenoinjertos.

El término osteoconductor implica la existencia de una matriz o sustrato tridimensional que facilite la migración, implantación y proliferación de las células mesenquimales, que se diferenciarán en células progenitoras óseas y formarán hueso ${ }^{(16)}$. Estas sustancias y materiales tienen la estructura microscópica similar al hueso esponjoso. Los autoinjertos, aloinjertos, xenoinjertos (o injertos óseos heterólogos obtenidos de alguna especie animal) y muchos injertos sintéticos tienen esta capacidad.

La osteoinducción consiste en la capacidad de reclutar esas células mesenquimales e inducir su diferenciación en células formadoras de hueso. La osteoinducción está mediada por factores o moléculas reguladoras del metabolismo óseo (BMP, PDGF, FGF, IGF, VEGF15) provenientes del injerto. Esta capacidad es escasa en los injertos mineralizados (cuya máxima capacidad osteoinductora proviene de las células vivas que portan) y muy importante en los no mineralizados. Un buen estado del lecho sobre el que asienta el injerto osteoinductor es clave para su éxito, ya que este depende del reclutamiento de células del huésped. Son sustancias osteoinductoras los injertos autólogos, aloinjertos desmineralizados y proteínas morfogenéticas obtenidas mediante técnicas de ingeniería genética (BMP, proteínas morfogenéticas del hueso)(17).

La capacidad osteogénica es la de incluir células indiferenciadas o progenitoras de aquellas capaces de formar hueso. Solo el hueso autólogo, y especialmente el esponjoso, tiene esta capacidad.

\section{Desventajas}

En los últimos años, han aumentado las publicaciones en las que se ponen de manifiesto las des- 
ventajas del uso de injerto autólogo, como consecuencia de las complicaciones asociadas ${ }^{(6,18,99)}$, lo que está llevando a la utilización, cada vez más frecuente, de aloinjertos y sustitutos del injerto óseo (alternativas ortobiológicas sintéticas)(20-22).

Las complicaciones más prevalentes y publicadas en la literatura son aquellas referidas a la cresta iliaca(18,23), aunque el dolor en el lugar de obtención del injerto, hematoma, infección, fractura, cicatriz hipertrófica y lesión nerviosa puede aparecer en cualquier lugar... ${ }^{(19)}$. Además, la cantidad de injerto óseo disponible es limitada y puede ser insuficiente para rellenar grandes defectos óseos. Como se verá más adelante, la calidad del hueso obtenido es variable en función del lugar de obtención.

\section{Tipos de injerto}

La decisión de uso o no de algún tipo de material óseo para injertar (esponjoso vs. estructural, local vs. a distancia, autoinjerto vs. aloinjerto) depende de la preferencia del cirujano.

\section{Cortical o estructural}

El injerto cortical óseo se suele utilizar para dar estabilidad mecánica inmediata en defectos estructurales, con el objetivo de restaurar la longitud, altura y/o alineamiento, o como material de interposición en las osteotomías de apertura. Tiene una capacidad osteoconductora moderada, con escasas propiedades osteoinductivas y osteogénicas. La matriz cortical tan densa permite una revascularización lenta y su incorporación al lugar donante, por lo tanto, también lo es. Así, durante los primeros 6 meses tras su implante estos injertos se van progresivamente debilitando (por la resorción ósea que ocurre previamente a la formación de hueso nuevo), para aumentar su resistencia en los siguientes 12 meses.

\section{Esponjoso}

El injerto esponjoso o no estructural es el más comúnmente obtenido. Se utiliza para las artrodesis in situ, relleno de pequeñas cavidades o irregularidades y en las pseudoartrosis con la intención de promover la consolidación ósea. Aporta una mayor superficie de contacto y mayor potencial celular, en comparación con el estructural, y su incorporación es rápida, en pocas semanas. Se sabe que a los 2 días está completamente vascularizado, en pocas semanas comienza la nueva formación ósea y que en 8 semanas el injerto ha sido completamente remodelado(24).

\section{Corticoesponjoso}

Tiene las ventajas de los anteriores: estabilidad estructural inmediata con las capacidades osteoinductivas y osteogénicas del hueso esponjoso. Este tipo de injerto se suele obtener de la cresta ilaca, que permite injerto uni-, bi- o tricortical.

\section{Injerto vascularizado}

Para incrementar la incorporación del injerto y la consolidación, podemos obtener injerto cortical o corticoesponjoso con un pedículo vascular (injerto vascularizado), indicado en defectos óseos importantes (más de 10-12 cm). Los más utilizados son los de cresta iliaca con la arteria circunfleja profunda, el peroné con arteria peroneal, radio distal con la arteria suprarretinacular y las costillas, con la arteria intercostal posterior. Según algún estudio, más del $90 \%$ de los osteocitos trasplantados sobrevive, haciendo a este tipo de injertos altamente osteogénicos ${ }^{(24)}$.

\section{Local o a distancia}

Injerto autólogo local es aquel obtenido mediante la misma incisión quirúrgica y distante es aquel obtenido por otra incisión separada.

Se han descrito en la literatura numerosos lugares de obtención de injerto autólogo en la cirugía de pie y tobillo, siendo los más comunes la cresta iliaca, el calcáneo, el peroné y la tibia proximal o distal ${ }^{(6,19,25-32)}$.

\section{Cresta iliaca}

La cresta iliaca anterior o posterior es frecuentemente utilizada como lugar de obtención de in- 


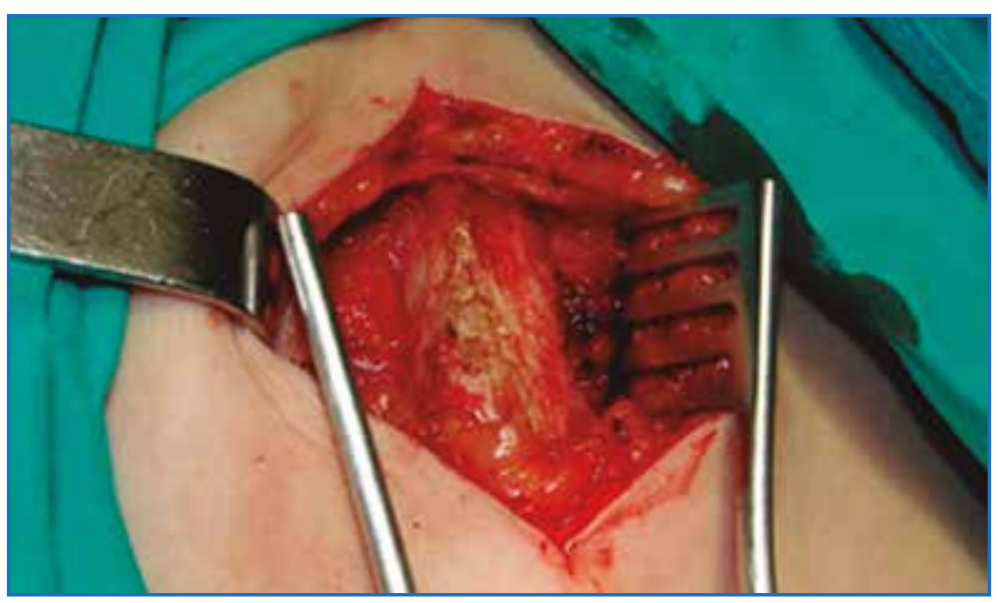

Figura 1. Exposición de cresta iliaca para obtención de injerto.

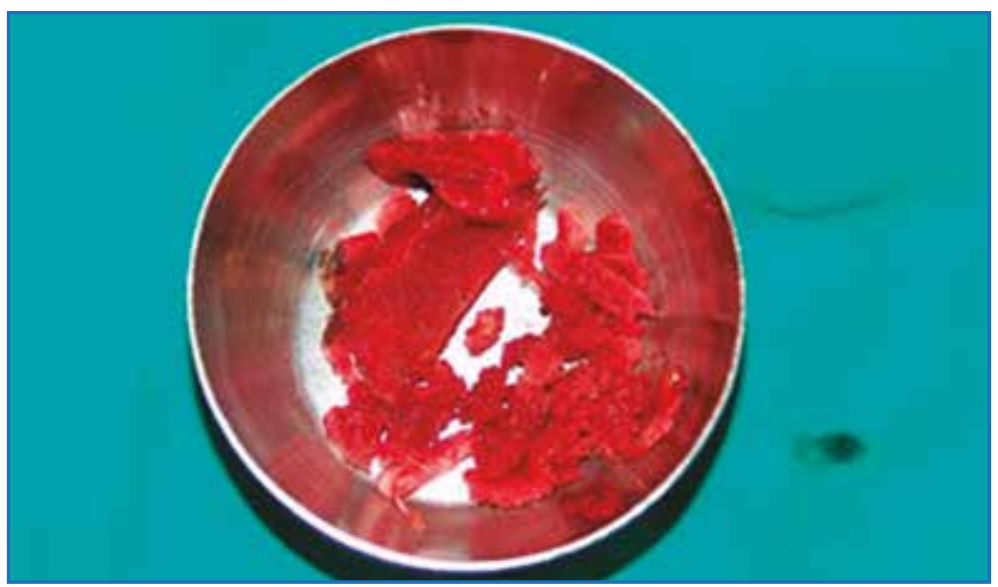

Figura 2. Injerto esponjoso obtenido de cresta iliaca.
Sin embargo, se han descrito hasta un $19,7 \%$ de complicaciones, incluyendo dolor en el lugar de toma de injerto, meralgia parestésica tras la lesión del nervio cutáneo femoral lateral, lesión de la arteria glútea superior, fractura de pelvis, hematoma, infección y alteraciones de la marcha ${ }^{(18,23)}$. Con la intención de minimizar estas complicaciones, se han descrito técnicas miniinvasivas $^{(33)}$ o percutáneas ${ }^{(34)}$.

\section{Tibia proximal y distal}

En las cirugías de pie y tobillo, la tibia proximal es una alternativa a considerar como sitio donante. Una de las ventajas principales es su proximidad anatómica. Las desventajas teóricas incluyen el volumen más limitado (comparado con la cresta iliaca) con $25 \mathrm{~cm}^{2}$ de media, las fracturas iatrogénicas y la menor calidad del hueso obtenido. Se ha publicado mucho al respecto en la literatura científica ${ }^{(25-29)}$.

Chiodo publica un estudio en el que compara las características biológicas del injerto autólogo de cresta iliaca con el de tibia proximal, concluyendo que existen diferencias significativas entre ambos, con respecto a la celularidad(35). Los injertos obtenidos de cresta iliaca

jerto autólogo debido a su facilidad de acceso y a la cantidad sustancial de hueso (desde $13 \mathrm{~cm}^{2}$ de la cresta anterior a $30 \mathrm{~cm}^{2}$ de la posterior) que es posible obtener, en comparación con otras localizaciones.

El injerto esponjoso o corticoesponjoso de cresta iliaca anterior se obtiene mediante una incisión paralela a la cresta iliaca, unos $3 \mathrm{~cm}$ posterior a la espina iliaca anterosuperior, evitando el nervio femorocutáneo (Figura 1). La cresta iliaca posterior se utiliza generalmente y por facilidad de acceso en cirugía de raquis.

La cresta iliaca aporta un sustrato osteoinductivo, osteoconductor y osteogénico, ya que su hueso trabecular contiene abundantes osteoblastos funcionales (Figura 2). Además, su amplia superficie de contacto favorece una rápida remodelación e incorporación. anterior contienen médula activa hematopoyéticamente, mientras que la grasa es predominante en los injertos tibiales con poca o nula actividad hematopoyética. Sin embargo, las tasas de consolidación son similares. Además, las complicaciones asociadas a la obtención de injerto de cresta iliaca, como se menciona previamente, pueden llegar hasta casi un $20 \%$, mientras que si se obtienen de tibia proximal rondan entre el 1,3 y el $3,4 \%{ }^{(18,23)}$.

\section{Peroné}

El peroné es un hueso tubular cortical, con escasa cantidad de esponjosa. Suele utilizarse como soporte estructural de forma clásica en artrodesis de tobillo abiertas, vía lateral o en empalizada en las últimas técnicas descritas ${ }^{(30,31)}$. 


\section{Calcáneo}

No hemos encontrado ningún artículo publicado en los últimos 10 años que analice la histología del injerto obtenido del cuerpo del calcáneo, aunque es de suponer que será similar al de otras metáfisis, como la tibia proximal o distal. Puede ser útil cuando se necesitan pequeñas cantidades.

\section{Factores de riesgo para el uso de injerto autólogo}

Mientras que históricamente las indicaciones de uso de injerto autólogo han sido, de alguna manera, paciente y cirujano dependientes, las elevadas tasas de complicaciones tanto en la obtención del injerto como las descritas en determinado grupo de pacientes tras las artrodesis quirúrgicas han servido como incentivo para definir mejor cuáles serían los factores de riesgo en los que sería recomendable el uso sistemático de injerto autólogo ${ }^{(14)}$.

Clásicamente, encontramos que esos factores de riesgo han sido los pacientes fumadores, intentos fallidos de fusión previos en otra articulación, necrosis avascular y diabetes, o, por supuesto, la presencia de una pseudoartrosis establecida(12).

Baumhauer et al. publican un estudio en el que encuestan a 66 cirujanos ortopédicos estadounidenses preguntándoles en qué condiciones utilizan injerto autólogo de hueso en las diferentes intervenciones de pie y tobillo(8). Los resultados los dividen en factores clínicos y factores radiográficos. Los factores clínicos por los que la mayoría de los cirujanos (> 90\%) utilizan injerto autólogo son la existencia actual de una pseudoartrosis, historia previa en ese paciente de no unión, fumadores, medicación concomitante que impida la consolidación ósea, deficiencia de vitamina $D$, diabetes e insuficiencia renal avanzada. Los factores radiográficos incluyen la evidencia radiográfica de no unión, necrosis avascular, aposición incongruente entre las superficies a artrodesar, pérdida ósea, osteoporosis, casos postraumáticos con colapso subcondral, quistes subcondrales, amplias superficies a artrodesar y la fusión de más de una articulación.

\section{Análisis de la literatura}

Hasta la fecha solo existen 2 metaanálisis o revisiones sistemáticas que sinteticen toda la información expuesta anteriormente y que nos puedan servir para la toma de decisiones en nuestro trabajo diario $0^{(7,11)}$.

En el artículo de Lareau et al. se revisan 159 artículos publicados sobre el uso de injerto autólogo en cirugía de pie y tobillo, desde 1959 hasta 2012, y se analiza, entre otras cosas, el nivel de evidencia científica ${ }^{(7)}$. Solo 2 de 159 tenía nivel 1 (grado de recomendación A) y 5 grado de evidencia II (grado de recomendación B) ${ }^{(21,22)}$. El resto eran series de casos retrospectivas, es decir, con nivel de evidencia científica IV y grado de recomendación C. Como conclusiones destacables de este estudio, cabe señalar que los autores afirman que se obtienen los mismos porcentajes de consolidación con el uso de injerto autólogo esponjoso (93,7\%), aloinjerto esponjoso (93,3\%), autoinjerto estructural $(94,2 \%)$ y sin injerto de ningún tipo (91\%)... sin diferencias estadísticamente significativas entre ningún grupo. Solo se obtiene un menor porcentaje de consolidación $(86,9 \%)$ cuando se utiliza aloinjerto estructural. En este último caso, destacan los autores, las tasas de consolidación no son comparables a los otros supuestos ya que, por lo general, se trata de cirugías mucho más complejas con gran pérdida ósea o colapso, con evidentes peores resultados. En cuanto a los factores de riesgo del paciente, aunque la mayoría de los cirujanos de pie y tobillo están de acuerdo en que el tabaco y la diabetes aumentan el riesgo de ausencia de consolidación, no hay estudios publicados que lo demuestren.

En el otro metaanálisis, Müller et al. ${ }^{(11)}$ analizan 10 publicaciones (958 retropiés) referidas al uso de injerto autólogo y sustitutos en las artrodesis y osteotomías de retropié, y tan solo encuentran 3 estudios prospectivos $(21,22,36)$. Estos autores concluyen que la evidencia actual sugiere que los aloinjertos estructurales son, al menos, no inferiores al autoinjerto en cuanto a los porcentajes de consolidación en artrodesis y osteotomías del retropié.

En el artículo publicado por Chiodo ${ }^{(35)}$ se pone de manifiesto que existen diferencias marcadas desde el punto de vista histológico entre los injertos óseos obtenidos de cresta iliaca y los de 
tibia proximal ${ }^{(35)}$. Sin embargo, en el mismo artículo destacan que el éxito clínico obtenido con el injerto de tibia proximal, a pesar de la mayor escasez de celularidad, pone en duda la necesidad de la capacidad osteogénica de autoinjerto para la consolidación. Concluyen finalmente que, en procedimientos primarios, el uso de autoinjerto puede ser sustituido por aloinjerto de esponjosa, con los mismos porcentajes de consolidación.

\section{Discusión}

La consolidación obtenida tras una fusión en pie y tobillo no solo se basa en la presencia o ausencia de injerto óseo, sino que depende de múltiples factores como la estabilidad mecánica (osteosíntesis rígida), las comorbilidades del paciente, la técnica quirúrgica, la complejidad del proceso, el cumplimiento del paciente y el protocolo postoperatorio. Además, existe un número identificable de factores que influyen en los porcentajes de consolidación y, pueden ser, además, factores de confusión y sesgo cuando analizamos los diferentes artículos.

A pesar de que existe un gran número de publicaciones que informan sobre los porcentajes de consolidación en pie y tobillo y las diferentes formas de uso del autoinjerto, sorprende las escasas conclusiones que pueden sacarse de un análisis exhaustivo de esta información. Aunque parece que el porcentaje de consolidación es discretamente superior (pero nunca alcanza el $100 \%$ ) en aquellos pacientes en los que se usa injerto autólogo, en los que no se utiliza ningún tipo de aporte supera el $90 \%$. Este dato a tener en cuenta es por lo que algunos autores no recomiendan su uso sistemático.

El injerto óseo autólogo tiene ventajas teóricas en cuanto a su capacidad osteoformativa, aunque 2 de los estudios nivel 1 demuestran que no existe tal diferencia entre el injerto autólogo y las alternativas ${ }^{(21,22)}$.

Las indicaciones específicas para el uso de algún tipo o cantidad de injerto también están pobremente documentadas. Solo parece claramente establecido el uso de injerto estructural para restaurar altura, alineación o longitud y el uso de hueso esponjoso para rellenar espacios, aumentar la aposición ósea y, teóricamente, aumentar la capacidad de consolidación.
Vista y analizada toda esta información, el énfasis científico actual se dirige hacia el desarrollo de alternativas biológicas que eliminen la necesidad de obtener injerto óseo para aumentar las fusiones óseas.

\section{Conclusiones}

Es difícil revisar sistemáticamente todos los artículos publicados en la literatura sobre el uso de injerto autólogo en las cirugías de pie y tobillo, ya que están llenos de factores de confusión. En la bibliografía disponible se incluye la experiencia de múltiples cirujanos, haciendo diferentes técnicas en diferentes articulaciones del pie y tobillo, en diferentes instituciones sanitarias, con pacientes muy variables, con multitud de indicaciones, y numerosos métodos de análisis de resultados.

Hay, además, varios factores conocidos que influyen en el porcentaje de consolidación por sí mismos y que necesitan ser analizados minuciosamente (sistemas de fijación, técnica quirúrgica, inmovilización postoperatoria...), especialmente en las cirugías de pie y tobillo, donde estos indices de consolidación son superiores al $90 \%$.

El injerto óseo autólogo continúa siendo, en la actualidad, el patrón oro (gold standard) en las cirugías en las que necesitemos aumentar los porcentajes de consolidación, aún no claramente definidas, debido a sus características biológicas, aunque se obtienen resultados solo discretamente superiores o iguales que con el uso de injerto alogénico o sin él, según algunos estudios.

No nos atrevemos a sentar indicaciones firmes con evidencia científica en las que sea imprescindible el uso de injerto autólogo, pero tal vez sean exclusivamente las ausencias de consolidación o pseudoartrosis, en las que sería recomendable la obtención de injerto de cresta iliaca. Para el resto de las indicaciones, son necesarios más estudios aparte de las series de casos u opinión de expertos que se recogen actualmente en la literatura.

\section{Bibliografía}

1. Pountos I, Giannoudis PV. Is there a role of coral bone substitutes in bone repair? Injury. 2016;47(12):2606-13.

2. Zhang G, Brion A, Willemin AS, Piet MH, Moby V, Bianchi $A$, et al. Nacre, a natural, multi-use and timely bioma- 
terial for bone graft substitution. J Biomed Mater Res A. 2017;105(2):662-71.

3. Panchbhavi, VK. Synthetic bone grafting in foot and ankle surgery. Foot Ankle Clin. 2010;15(4):559-76.

4. Mowlem, R. Cancellous chip bone grafts: Report of 75 cases. Lancet. 1944;2:746-8.

5. Burchardt $H$. The biology of bone graft repair. Clin Orthop Relat Res. 1983;74:28-42.

6. Myeroff C, Archdeacon M. Autogenous bone graft: donor sites and techniques. J Bone Joint Surg Am. 2011;93(23):2227-36.

7. Lareau CR, Deren ME, Fantry A, Donahue RM, DiGiovanni CW. Does autogenous bone graft work? A logistic regression analysis of data from 159 papers in the foot and ankle literature. Foot Ankle Surg. 2015;21(3):150-9.

8. Baumhauer JF, Pinzur MS, Daniels TR, Lin SS, Beasley W, Donahue RM, et al. Survey on the need for bone graft in foot and ankle fusion surgery. Foot Ankle Int. 2013;34(12):1629-33.

9. Myerson MS, Filippi J. Interphalangeal joint lengthening arthrodesis for the treatment of the flail toe. Foot Ankle Int. 2010;31(10):851-6.

10. Templin D, Jones $K$, Weiner DS. The Incorporation of Allogeneic and Autogenous Bone Graft in Healing of Lateral Column Lengthening of the Calcaneus. Foot Ankle Surg. 2008;47(4):283-7.

11. Müller MA, Frank A, Briel M, Valderrabano V, Vavken $P$, Entezari $V$, et al. Substitutes of structural and non-structural autologous bone grafts in hindfoot arthrodeses and osteotomies: a systematic review. BMC Musculoskelet Disord. 2013;14:59.

12. Ishikawa SN, Murphy GA, Richardson EG. The effect of cigarette smoking on hindfoot fusions. Foot Ankle Int. 2002;23(11):996-8.

13. Easley ME, Trnka HJ, Schon LC, Myerson MS. Isolated subtalar arthrodesis. J Bone Joint Surg Am. 2000;82(5):613-24.

14. Frey C, Halikus NM, Vu-Rose T, Ebramzadeh E. A review of ankle arthrodesis: predisposing factors to nonunion. Foot Ankle Int. 1994;15(11):581-4.

15. Haddad SL, Coetzee JC, Estok R, Fahrbach K, Banel D, Nalysnyk L. Intermediate and long-term outcomes of total ankle arthroplasty and ankle arthrodesis. A systematic review of the literature. J Bone Joint Surg Am. 2007;89(2):1899-905.

16. Cornell CN, Lane JM. Current understanding of osteoconduction in bone regeneration. Clin Orthop Relat Res. 1998;355(Suppl):267-73.

17. Miron RJ, Zhang Q, Sculean A, Buser D, Pippenger $\mathrm{BE}$, Dard $\mathrm{M}$, et al. Osteoinductive potential of $4 \mathrm{com}$ - monly employed bone grafts. Clin Oral Investig. 2016;20(8):2259-65.

18. Dimitriou R, Mataliotakis Gl, Angoules AG, Kanakaris NK, Giannoudis PV. Complications following autologous bone graft harvesting from the iliac crest and using the RIA: a systematic review. Injury. 2011;42(Suppl 2):3-15.

19. Baumhauer J, Pinzur MS, Donahue R, Beasley W, DiGiovanni C. Site selection and pain outcome after autologous bone graft harvest. Foot Ankle Int. 2014;35(2):104-7.

20. Finkemeier CG. Bone-grafting and bone-graft substitutes. J Bone Joint Surg Am. 2002;84(3):454-64.

21. Digiovanni CW, Baumhauer J, Lin SS, Berberian WS, Flemister AS, Enna MJ, et al. Prospective: randomized, multi-center feasibility trial of rhPDGF-BB versus autologous bone graft in a foot and ankle fusion model. Foot Ankle Int. 2011;32(4):344-54.

22. Dolan CM, Henning JA, Anderson JG, Bohay DR, Kornmesser MJ, Endres TJ. Randomized prospective study comparing tri-cortical iliac crest autograft to allograft in the lateral column lengthening component for operative correction of adult acquired flatfoot deformity. Foot Ankle Int. 2007;28(1):8-12.

23. DeOrio JK, Farber DC. Morbidity associated with anterior iliac crest bone grafting in foot and ankle surgery. Foot Ankle Int. 2005;26(2):147-51.

24. Khan SN, Cammisa FP Jr, Sandhu HS, Diwan AD, Girardi FP, Lane JM. The biology of bone grafting. J Am Acad Orthop Surg. 2005;13(1):77-86.

25. O'Malley DF Jr, Conti SF. Results of distal tibial bone grafting in hindfoot arthrodeses. Foot Ankle Int. 1996;17(7):374-7.

26. Alt V, Meeder PJ, Seligson D, Schad A, Atienza C Jr. The proximal tibia metaphysis: a reliable donor site for bone grafting? Clin Orthop Relat Res. 2003;41(4):315-21.

27. Geideman W, Early JS, Brodsky J. Clinical results of harvesting autogenous cancellous graft from the ipsilateral proximal tibia for use in foot and ankle surgery. Foot Ankle Int. 2004;25(7):451-5.

28. O'Keeffe RM Jr, Riemer BL, Butterfield SL. Harvesting of autogenous cancellous bone graft from the proximal tibial metaphysis. A review of 230 cases. J Orthop Trauma. 1991;5(4):469-74.

29. Whitehouse MR, Lankester BJA, Winson IG, Hepple S. Bone graft harvest from the proximal tibial in foot and ankle arthrodesis surgery. Foot Ankle Int. 2006;27(11):913-6.

30. leong E, Mahapatra P, Nathan S. Fashioning autologous bone graft from the fibula in the trasfibular approach to open ankle arthrodesis. Foot Ankle Surg. 2014;20(2):149-50. 
31. Paul J, Barg A, Horisberger $M$, Herrera $M$, Henninger $\mathrm{HB}$, Valderrabano V. Ankle salvage surgery with autologous circular pillar fibula augmentation and intramedullary hindfoot nail. I Foot Ankle Surg. 2014;53(5):601-5.

32. Singleton TJ, Schuberth JM. Bone graft from the distal medial tibia in foot and ankle surgery. Foot Ankle Spec. 2012;5(3):168-74.

33. Kane JM, Raikin SM. Surgical tip: a minimally invasive mini open technique for harvesting iliac crest bone graft. Foot Ankle Int. 2013;34(8):1182-4.
34. Hernigou P, Guissou I, Homma Y, Poignard A, Chevallier $\mathrm{N}$, Rouard $\mathrm{H}$, et al. Percutaneous injection of bone marrow mesenchymal stem cells for ankle non-unions decreases complications in patients with diabetes. Int Orthop. 2015;39(8):1639-43.

35. Chiodo CP, Hahne J, Wilson MG, Glowacki J. Histological differences in iliac and tibial bone graft. Foot Ankle Int. 2010;31(5):418-22.

36. Michelson JD, Curl LA. Use of demineralized bone matrix in hindfoot arthrodesis. Clin Orthop Relat Res. 1996;(325):203-8. 